Objective: To analyze the macronutrient content of infant and toddler foods (ITF) containing vegetables in the United States.

Study Design: Company websites $(n=19)$ were used to create a database of commercial ITF's containing vegetables $(n=517)$. The following information was recorded for each product: name, ingredients, serving size (g), total calories, carbohydrates (g), and sugars (g) per serving. Ingredient lists were used to categorize products into the following groups: single vegetable $(n=48)$, multiple vegetable $(n=40)$, and vegetable/fruit $(n=150)$. Remaining products containing proteins or grains were excluded from this analysis.

Outcome Measures and Analysis: Analyses of Variance (ANOVAs) were used to compare the macronutrient content per serving across vegetable product categories.

Results: ANOVAs showed significant differences among the vegetable products for total calories, $\mathrm{F}(2,235)=$ $13.74, \mathrm{p}<.001)$, carbohydrates, $\mathrm{F}(2,235)=29.46, \mathrm{p}<$ $.001)$, and sugars per serving, $\mathrm{F}(2,235)=94.05, \mathrm{p}<$ $.001)$. Post-hoc tests revealed significant differences between the single and multiple vegetable products separately and the vegetable/fruit products for total carbohydrates and sugars (Ps $<.001)$. However, the single and multiple vegetable products did not differ significantly from one another on any macronutrient (Ps $>.05$ ).

Conclusions and Implications: Exposure to vegetable flavors during infancy increases the likelihood that these flavors will be accepted during later childhood. However, our data reveal that a substantial majority of commercial infant and toddler vegetable products contain fruits. The addition of fruits to vegetable products affects the flavors infants and toddlers are exposed to, and significantly increases the carbohydrates and simple sugars per serving.

Funding: None

\section{P180 WITHDRAWN}

\section{P181 Online ASA24 Training Manual Pilot- Tested with Expanded Food and Nutrition Education Program (EFNEP) Educators}

Lori Anderson Spruance, PhD, CHES, Brigham Young University; Deirdre Douglass, $M S, R D$, Westat;

Thea Palmer Zimmerman, MS, RD;

Patricia M. Guenther, PhD, RD, University of Utah; Karen Franck, PhD, University of Tennessee; Debie Head, EdD, RD, LD, CDE, University of Arkansas Division of Agriculture Cooperative Extension Service; Teresa Henson, BS; Nathan Millerberg, BS, Utah State University; Carla J. Moore, PhD, RD, University of Georgia; Kimberly Wilson-Sweebe, MS, South Dakota State University; Gina Wood, MPH, RD, LD, West Virginia University; Carrie M. Durward,PhD, RD, carrie.durward@ usu.edu, Utah State University, 8700 Old Main Hill, Logan, UT 84321

Objective: The purpose of this study was to evaluate an online training manual designed to teach paraprofessionals how to use the mobile version of the Automated Self-Administered 24-hour recall (ASA24-2016) to collect 24-hour dietary intake data.

Study Design, Setting, Participants: Nutrition educators from EFNEP programs in 17 states were recruited to complete an online training consisting of readings, videos, online activities, quizzes, and assisting two individuals in completing a dietary recall using ASA24. After completing the training, the educators were invited to complete a onetime online survey.

Outcome Measures and Analysis: The questionnaire assessed the training content, reading and comprehension level, and appearance and design of the manual. Descriptive results were summarized for each domain.

Results: Twenty-five educators completed the online training and 14 (56\%) completed the online questionnaire. Most of the survey respondents took 4-7 hours to complete the training $(9 ; 64 \%)$. The majority identified as White $(11 ; 79 \%)$ and female $(13 ; 93 \%)$. The majority of respondents $(8 ; 57 \%)$ utilized both the 
P181 (continued)

readings and videos to complete the training and most $(11 ; 79 \%)$ felt the combination of both was most effective for learning. After completing the training, 11 (79\%) of respondents felt prepared to collect data using ASA24-2016.

Conclusions and Implications: Respondents had positive feedback for the content, reading level, appearance and design, and activities of the training. Results from this study suggest that the training is effective in preparing EFNEP nutrition educators to collect 24-hour dietary recall data using ASA24-2016; although potential bias due to self-selection and non-response is a concern. The feedback provided will inform future improvements to the manual.

Funding: USDA

\section{P182 Outcome Evaluation of a School-Based Intervention Implemented by Maine SNAP-Ed}

Brenda Wolford, MS, RD, brenda.wolford@altarum.org, Altarum Institute, 4 Milk Street, 3rd Floor, Portland, ME 04101; Rebecca Harnik, MS, Altarum Institute; Brent Walker, MS, RD; Margaret Wilkin, MPH; Patricia Dushuttle, MA, State of Maine DHHS Office for Family Independence; Elizabeth Pratt, MPH, University of New England; Pamela Bruno, MPH; Kira Rodriguez, MHS

Objective: To compare changes over time in fruit and vegetable consumption of students receiving a multi-level nutrition education intervention.

Theory, Prior Research, Rationale: Maine SNAP-Ed delivers evidence-based interventions in low-income communities supported by policy, systems and environmental (PSE) change strategies. Maine's SNAP-Ed implementing agency, the University of New England, selected Pick a Better Snack (PABS) for use in elementary schools. PABS, developed by the Iowa Nutrition Network, was previously evaluated and shown to significantly impact child fruit and vegetable consumption.

Description: PABS offers monthly lessons, take-home materials for parents and leave-behind classroom lessons. Supported by PSE strategies, PABS focuses on improving dietary intake and promoting physical activity. Target audience were second and third grade students and their parents/caregivers in eligible schools receiving the intervention.

Evaluation: Altarum conducted an evaluation of 222 families using pre/post-tests, and UNE conducted a postonly evaluation of classroom teachers.

Conclusions and Implications: Following the intervention, the evaluation revealed increases in frequency of children asking for fruits and vegetables and eating multiple kinds of fruits; and in parents/caregivers offering fruits and vegetables. After indirect education, parents/caregivers increased their own fruit and vegetable consumption. Take-home materials had a significant association with healthy eating; the child's consumption of fruits and vegetables increased if the parent reported using the information sent home. These findings reinforce the importance of extending nutrition education to teachers and parents. Additionally, PSE work in settings where PABS has been offered may help sustain positive changes. PABS appears to be an effective intervention to improve dietary intake among low-income families in Maine.

Funding: Supplemental Nutrition Assistance Program Education

\section{P183 Outcome Evaluation of Maine SNAP-Ed's Print and Digital Social Marketing Campaign}

Brent Walker, MS, RD, brent.walker@altarum.org, Altarum Institute, 4 Milk Street, 3rd Floor, Portland, ME 04101; Brenda Wolford, MS, $R D$, Altarum Institute; Patricia Dushuttle, MA, State of Maine DHHS Office for Family Independence

Objective: To determine effectiveness of reaching Maine SNAP-Ed's target audience with social marketing messages delivered through print and digital channels.

Theory, Prior Research, Rationale: USDA's Food and Nutrition Service (FNS) encourages the use of social marketing campaigns as a way to enhance nutrition education in SNAP-Ed. Successful campaigns are comprehensive in scope and provide education through multiple channels.

Description: In 2015, Maine SNAP-Ed launched its campaign, called Shop, Cook, Eat Healthy and on a Budget, to influence health-related behaviors of individuals and communities while building upon direct nutrition education efforts. The campaign integrated traditional print materials (direct mail postcards, posters, flyers), with a robust online presence (targeted online ads, social media, landing page). Target audience were SNAP-eligible women with children in Maine.

Evaluation: Altarum conducted a follow-up randomized phone survey of 600 SNAP recipients statewide using measures from the SNAP-Ed Evaluation Framework to assess campaign exposure. Awareness of the campaign increased significantly from the previous year ( $63 \%$ vs. $49 \%)$, and women with children reported higher levels of exposure to messages compared to other respondents ( $81 \%$ vs. $56 \%)$, indicating that messages are resonating with the target audience. While print materials were the most commonly recalled among all respondents, women with children had higher levels of recall of the digital campaign compared to others. Exposure to the campaign led many respondents to report tying out a new behavior such as making healthier food choices (32\%).

Conclusions and Implications: Evaluation results indicate that Maine SNAP-Ed has designed a social marketing campaign that reaches a large segment of its target audience through print and digital channels.

Funding: Supplemental Nutrition Assistance Program Education 